\title{
Editorial
}

\section{Antibody-based targeted therapy for gastric cancer}

\author{
Florian Lordick, Christian Peschel, and Jörg Rüdiger Siewert \\ Klinikum rechts der Isar, Technical University of Munich, Ismaninger Str. 22, D-81675 Munich, Germany
}

Systemic chemotherapy has been used to treat gastric cancer in Japan, Europe, and North America since the late 1950s. However, the benefit remained unclear until the 1990s, when several studies revealed a quantifiable improvement of life expectancy and quality of life following chemotherapy in patients with stage IV disease. Unfortunately, prognosis remains very poor in patients with advanced disease. In large-scale, prospectively controlled randomized phase III trials, median survival times for patients with metastatic disease do not generally exceed 10 months [1]. In stage II and III disease, the use of perioperative chemotherapy, and also radiotherapy, is still controversial [2].

One of the key treatment issues is that gastric cancer has limited chemosensitivity. While chemotherapy can result in infrequent complete remissions, partial responses are usually of very short duration and many of the treatment regimens in use are cumbersome to administer and can cause serious side effects. In brief, effective systemic treatment for patients with advanced gastric cancer is currently an unmet need in medical oncology.

Over the past few years, research into tumor biology, the cellular and molecular mechanisms responsible for malignant proliferation and tumor spread, and increasing knowledge of specific targets has resulted in the development of surprisingly active anti-tumor drugs. Of these, monoclonal antibodies (mAbs), directed against specific cell-surface antigens or their ligands, represent the most widely used and effective "targeted therapies" in the treatment of malignant tumors. Bevacizumab (Avastin; Genentech, San Francisco, CA, USA), a recombinant humanized $\mathrm{mAb}$ targeted against vascular epithelial growth factor (VEGF), is approved for the treatment of metastatic colorectal cancer, based on data showing improved survival when added to chemotherapy $[3,4]$. Bevacizumab can also augment the activ-

Offprint requests to: F. Lordick ity of chemotherapy in a variety of other solid tumors [5].

Several mAbs targeting members of the epidermal growth factor receptor (EGFR) family have also been developed [6]. The human epidermal growth factor receptor-1 (HER1) is a transmembrane glycoprotein that has intrinsic tyrosine kinase activity. In its activated form it fuels the malignant properties of many tumors via a multistep phosphorylation process involving various downstream signaling cascades. Cetuximab (Erbitux; ImClone, New York, NY, USA), a chimeric human/mouse IgG1 mAb, has been shown to be active in metastatic colorectal cancer and head and neck tumors. It enhances the activity of chemotherapy and radiotherapy. Other EGFR/HER1-targeted therapies include nimotuzumab (TheraCIM, a chimeric IgG1 $\mathrm{mAb}$ ) and matuzumab (EMD 72000, a humanized IgG1 $\mathrm{mAb}$ ), as well as panitumumab (ABX-EGF, a fully human $\mathrm{IgG} 2 \mathrm{mAb}$ ). All three $\mathrm{mAbs}$ are under clinical investigation in different solid tumors.

A comparable mechanism of action applies for the humanized IgG1 mAb trastuzumab (Herceptin; Genentech), which targets the HER2 protein, another member of the EGFR family. Trastuzumab has shown considerable efficacy in the palliative treatment of HER2-positive metastatic breast cancer. It has also been evaluated in the adjuvant treatment of early-stage breast cancer, where it appears to improve survival outcomes in women with resected disease. Now that we are able to improve survival in patients with novel "targeted therapies" in both metastatic colorectal cancer (with bevacizumab) and breast cancer (with trastuzumab), the search for targeted agents that could offer similar benefits in gastric cancer is gaining momentum.

The first and most important step in the development of targeted treatment in gastric cancer is to determine whether the potential target is overexpressed or found in a particularly activated form and if this has a specific impact on growth dynamics or tumor spread. VEGF is a 
dimeric, heparin-binding glycoprotein that functions as a potent mitogen for vascular endothelial cells, promoting their migration and organization for the neovascularization of micrometastases. In gastric carcinomas, VEGF tumor expression and serum levels have been positively correlated with vascular involvement, and with lymph node, liver, and peritoneal metastases $[7,8]$. Moreover, patients with VEGF-positive tumors have a poorer prognosis than those with VEGFnegative tumors [7,8]. Researchers at the Memorial Sloan Kettering Cancer Center are currently evaluating bevacizumab in combination with irinotecan and cisplatin in a phase II study of patients with gastric and gastroesophageal junction cancers (NCT00084604). Preliminary results are promising, but a high rate of treatment-related thromboembolic events has been observed and merits close attention in these patients [9].

The HER1 receptor of the EGFR family is overexpressed in $10 \%-50 \%$ of gastric cancer patients. Overexpression of EGFR, as determined by immunohistochemistry (IHC), almost exclusively occurs by gene amplification and is associated with shorter survival $[10,11]$. Unfortunately, the IHC level of EGFR expression does not appear to hold promise as an appropriate marker for predicting response in patients receiving anti-EGFR mAbs. Therefore, a critical question is: who will benefit from treatment against EGFR [12]? To find an answer, all clinical trials employing anti-EGFR mAbs need to encompass translational research programs. Ideally, these should comprise an analysis of EGFR activity and of downstream targets (PI3K, Akt, and MAPK) by IHC, detection of EGFR mRNA expression by real-time quantitative reverse transcriptase-polymerase chain reaction (RT-PCR) analysis, detection of EGFR gene amplification by fluorescence in situ hybridization (FISH), detection of EGFR mutations by sequence and wave analysis, and some method of correlating EGFR expression, activity, mutation, and amplification with therapeutic response and clinical benefit. Mutations of the EGFR receptor that have been found to be associated with response and survival in the treatment of non-small-cell lung cancer with the tyrosine-kinase inhibitor gefitinib [13] have never been shown to be predictive when EGFRtargeting antibodies were applied. This observation can be interpreted in favor of therapy with monoclonal EGFR-antibodies, because a broader spectrum of activity may be expected. Studies assessing the activity of anti-HER1 mAbs in advanced gastric and esophagogastric cancer are now underway, including a German multicenter trial combining cetuximab with weekly oxaliplatin and 5-fluorouracil/leucovorin (5-FU/LV; AIO STOMOX-2 trial). Another phase II trial, at the Royal Marsden Cancer Centre in the United Kingdom, is evaluating matuzumab with epirubicin/cisplatin plus capecitabine chemotherapy (NCT00113581).

Studies of HER2-positivity rates in gastric cancer using IHC have shown a mean value of approximately $18 \%$, when positivity was defined as either IHC $3+$ or equivalent strong overexpression. Studies using FISH or chromogenic in situ hybridization (CISH) have shown a mean value of approximately $19 \%$. Interestingly, gene amplification seems to vary considerably between different histological subtypes, with $22 \%$ of cancers of the intestinal type according to Lauren being overexpressed, compared with a much lower degree of expression in non-intestinal tumors [14]. However, this finding has not been confirmed by Japanese investigators [15], who reported that the presence of HER2 amplification was associated with poor carcinomaspecific survival.

In the study by Tanner et al. [14], the anti-HER2 $\mathrm{mAb}$ trastuzumab inhibited the growth of HER2positive gastric cancer cell lines in vitro and in tumor xenografts. Interestingly, co-amplification of topoisomerase II $\alpha$ was present in the majority of gastric cancers with HER2 amplification [14]. This latter finding might encourage clinical investigations to use topoisomerase II $\alpha$ inhibitors such as anthracyclines or etoposide in conjunction with trastuzumab in this particular group of patients. An international randomized multicenter phase III trial (BO18255) has recently been initiated to investigate the role for trastuzumab in HER2-positive (IHC or FISH) adenocarcinomas of the stomach or the gastroesophageal junction.

After the exciting developments in breast, colon, lung, and other cancer types over the past few years, it seems probable that targeted therapies will also prove effective and find their place in the treatment of gastric cancer. However, the journey from bench to bedside is often a long one. Our current understanding of the biological characteristics of gastric cancer does not allow us to predict which of the currently available therapeutic tools might be beneficial in the clinic. In this context, case reports like the one by Rebischung et al. [16], published in this issue of Gastric Cancer, are helpful. While a case report can never answer the question of the effectiveness of a new therapeutic approach in general, it can help to generate a hypothesis. The message from this case report is that the concurrent use of the anti-HER2 $\mathrm{mAb}$ trastuzumab may enhance the cytotoxic effects of chemotherapy in patients with HER2positive gastric cancer. The patient achieved a complete response when a third-line chemotherapy regimen was combined with trastuzumab. Of note, this remission lasted for 18 months with trastuzumab alone, indicating that the use of antibodies directed against the EGFR family may lead to markedly sustained remissions in gastric cancer, at least in subsets of patients. Therefore, 
this case report supports the rationale for current clinical investigations using $\mathrm{mAbs}$ in the treatment of gastric cancer.

In the light of the growing options we have in gastric cancer, not only in the palliative treatment of metastatic disease but also in the perioperative setting, emphasis should be placed on the identification of subgroups of patients who would most likely benefit from targeted therapies. Meanwhile, we eagerly await the findings from ongoing trials of these novel agents in this difficultto-treat disease.

\section{References}

1. Wagner AD, Grothe W, Behl S, Kleber G, Grothey A, Haerting $\mathrm{J}$, et al. Chemotherapy for advanced gastric cancer. Cochrane Database Syst Rev 2005;18:CD004064.

2. Lordick F, Siewert JR. Recent advances in multimodal treatment for gastric cancer: a review. Gastric Cancer 2005;8:78-85.

3. Kabbinavar F, Hurwitz HI, Fehrenbacher L, Meropol NJ, Novotny WF, Lieberman G, et al. Phase II, randomized trial comparing bevacizumab plus fluorouracil (FU)/leucovorin (LV) with FU/LV alone in patients with metastatic colorectal cancer. J Clin Oncol 2003;21:60-5.

4. Hurwitz H, Fehrenbacher L, Novotny W, Cartwright T, Hainsworth J, Heim W, et al. Bevacizumab plus irinotecan, fluorouracil, and leucovorin for metastatic colorectal cancer. N Engl J Med 2004;350:2335-42.

5. Midgley R, Kerr D. Bevacizumab - current status and future directions. Ann Oncol 2005;16:999-1004.

6. Baselga J, Arteaga CL. Critical update and emerging trends in epidermal growth factor receptor targeting in cancer. J Clin Oncol 2005;23:2445-59.
7. Maeda K, Chung YS, Ogawa S, Kang SM, Ogawa M, Sawada T, et al. Prognostic value of vascular endothelial growth factor expression in gastric carcinoma. Cancer 1996;77:858-63.

8. Yoshikawa T, Tsuburaya A, Kobayashi O, Sairenji M, Motohashi $\mathrm{H}$, Yonoma S, et al. Plasma concentrations of VEGF and bFGF in patients with gastric carcinoma. Cancer Lett 2000;153:7-12.

9. Shah MA, Ilson D, Kelsen DP. Thromboembolic events in gastric cancer: high incidence in patients receiving irinotecan- and bevacizumab-based therapy. J Clin Oncol 2005;23:2574-6.

10. Gamboa-Dominguez A, Dominguez-Fonseca C, QuintanillaMartinez L, Reyes-Gutierrez E, Green D, Angeles-Angeles A, et al. Epidermal growth factor receptor expression correlates with poor survival in gastric adenocarcinoma from Mexican patients: a multivariate analysis using a standardized immunohistochemical detection system. Mod Pathol 2004;17:579-84.

11. Takehana T, Kunitomo K, Suzuki S, Kono K, Fujii H, Matsumoto Y, Ooi A. Expression of epidermal growth factor receptor in gastric cancer. Clin Gastroenterol Hepatol 2003;1:438-45.

12. Arribas J, Baselga J. Who will benefit from treatment against EGFR? Lancet Oncol 2005;6:279-86.

13. Lynch TJ, Bell DW, Sordella R, Gurubhagavatula S, Okimoto RA, Brannigan BW, et al. Activating mutations in the epidermal growth factor receptor underlying responsiveness of non-smallcell lung cancer to gefitinib. N Engl J Med 2004;350:2129-39.

14. Tanner M, Hollmén M, Junttila TT, Kapanen AI, Tommola S, Soini Y, et al. Amplification of Her-2 in gastric carcinoma: association with topoisomerase II $\alpha$ gene amplification, intestinal type, poor prognosis and sensitivity to trastuzumab. Ann Oncol 2004; $16: 273-8$.

15. Takehana T, Kunimoto K, Kono K, Kitahara F, Iizuka H, Matsumoto Y, et al. Status of c-erbB-2 in gastric adenocarcinoma: a comparative study of immunohistochemistry, fluorescence in situ hybridization and enzyme-linked immuno-sorbent assay. Int $\mathbf{J}$ Cancer 2002;98:833-7.

16. Rebischung C, Barnoud R, Stéfani L, Faucheron J-L, Mousseau $M$. The effectiveness of trastuzumab (Herceptin) combined with chemotherapy for gastric carcinoma with overexpression of the cerbB-2 protein. Gastric Cancer 2005;8:249-252. 